\title{
Campylobacter jejuni proteins Cj0952c and Cj0951c affect chemotactic behaviour towards formic acid and are important for invasion of host cells
}

\author{
A. Malik Tareen,† Javid Iqbal Dasti,† Andreas E. Zautner, Uwe Groß \\ and Raimond Lugert \\ University Medical Center Göttingen, Institute for Medical Microbiology, Kreuzbergring 57, \\ 37075 Göttingen, Germany
}

Correspondence

Raimond Lugert

rlugert@gwdg.de

\begin{abstract}
Campylobacter jejuni, an important food-borne bacterial pathogen in industrialized countries and in the developing world, is one of the major causes of bacterial diarrhoea. To identify genes which are important for the invasion of host cells by the pathogen, we screened altogether 660 clones of a transposon-generated mutant library based on the clinical C. jejuni isolate B2. Thereby, we identified a clone with a transposon insertion in gene cj0952c. As in the well-characterized C. jejuni strain NCTC 11168, the corresponding protein together with the gene product of the adjacent gene $c j 0951 c$ consists of two transmembrane domains, a HAMP domain and a putative MCP domain, which together are thought to act as a chemoreceptor, designated Tlp7. In this report we show that genes cj0952c and cj0951c (i) are important for the host cell invasion of the pathogen, (ii) are not translated as one protein in C. jejuni isolate B2, contradicting the idea of a postulated read-through mechanism, (iii) affect the motility of $C$. jejuni, (iv) alter the chemotactic behaviour of the pathogen towards formic acid, and (v) are not related to the utilization of formic acid by formate dehydrogenase.
\end{abstract}

Revised 23 June 2010

Accepted 20 July 2010

\section{INTRODUCTION}

Campylobacter jejuni is a Gram-negative, spiral-shaped bacterium which is a major cause of bacterial diarrhoea in both developing and industrialized countries (Altekruse et al., 1999; Friedman et al., 2000). The infection is also characterized by fever and abdominal cramps, and in rare cases Guillain-Barré syndrome can emerge as a postinfection complication (Allos, 2001).

Despite its importance as a human pathogen, little is known about the mechanisms by which $C$. jejuni causes disease, although several publications have described potential virulence factors (Dasti et al., 2010). Putative adhesion factors have been identified, e.g. the fibronectinbinding proteins CadF and FlpA (Konkel et al., 1997, 2010), the autotransporter CapA, and a surface-exposed lipoprotein, JIpA (Jin et al., 2001). In addition, the sialylated lipooligosaccharide outer core of $C$. jejuni has been demonstrated to be an important factor for the invasion of epithelial cells (Guerry et al., 2000; Louwen et al., 2008). Moreover, C. jejuni synthesizes a set of proteins which are secreted by the flagellar export apparatus during co-culture of the pathogen with epithelial cells, and

†These authors contributed equally to this work.

Abbreviation: MCP, methyl-accepting chemotaxis protein. these are referred to as Campylobacter invasion antigens (Cia proteins) (Konkel et al., 1999). Although the function of these proteins is still unknown, mutation of $c i a B$ results in a significant reduction of competency to invade host cells (Konkel et al., 2004).

Motility of C. jejuni has been shown to be an intestinal colonization factor (Morooka et al., 1985), and O-linked glycosylation of flagellin is crucial for the attachment of the bacterium to intestinal epithelial cells (Yao et al., 1994). In addition, the motility of the pathogen is linked to chemotaxis, which was initially described by Hugdahl et al. (1988). In the genome sequence of C. jejuni NCTC 11168 , orthologues of the chemotaxis genes cheA, cheW, cheV, cheY, cheR and cheB have been identified, in which cheA encodes a histidine protein kinase activated by chemoreceptors. CheW interacts with the histidine kinase CheA, mediating the signal from the receptor, while CheY represents a chemotaxis regulator that interacts with the flagellar motor in a phosphorylated form. Furthermore, the proteins $\mathrm{CheR}$ and $\mathrm{CheB}$ serve as methyltransferase and methylesterase, respectively, for reversible chemoreceptor methylation (Hazelbauer et al., 2008). Finally, the presence of two aerotaxis genes and 10 putative chemoreceptor genes, designated Tlps for transducer-like proteins, has been revealed. These chemoreceptors can be further 
classified into different groups based on structural homologies (Marchant et al., 2002). Thereby, group A receptors possess a similar structure to methyl-accepting chemotaxis proteins (MCPs) of Escherichia coli and to family A transducers of Halobacterium salinarum (Zhang et al., 1996). Due to the presence of both transmembrane domains and a periplasmic ligand-binding domain, these group A receptors are thought to sense external ligands.

Performing BLASTX analysis, C. jejuni genes cj0952c and cj0951c are suggested to be pseudogenes in C. jejuni strain NCTC 11168, and it is thought that a read-through from upstream $c j 0952 c$ into downstream cj0951c would create one functional chemoreceptor, termed Tlp7. The background of this assumption is that Cj0952c possesses two transmembrane domains followed by a C-terminal HAMP domain, whereas Cj0951c is a putative MCP-domain signal transduction protein, and together they would constitute a typical group A receptor protein of a chemotaxis system (Marchant et al., 2002).

In this report, we describe the influence of the $C$. jejuni genes $c j 0952 c$ and $c j 0951 c$ on the ability of the pathogen to invade host cells. We also demonstrate that the gene products are not translated into a single protein, and consequently the corresponding genes cannot be characterized as pseudogenes in the clinical isolate B2. Furthermore, we demonstrate that these proteins act as a receptor that affects chemotaxis of the pathogen in the presence of formic acid and describe how the oxidation of formic acid by formate dehydrogenase does not depend on the presence of this chemoreceptor.

\section{METHODS}

Bacterial stains, media and culture conditions. The $C$. jejuni strains used in this study were B2, which was isolated in the University Medical Center Göttingen from a patient suffering from gastroenteritis (Schmidt-Ott et al., 2005; Dasti et al., 2007), and the C. jejuni strains 81-176 and NCTC 11168 . Bacteria were routinely grown on Columbia blood agar supplemented with $5 \%$ defibrinated sheep blood under microaerophilic conditions $\left(85 \% \mathrm{~N}_{2}, 10 \% \mathrm{CO}_{2}, 5 \%\right.$ $\mathrm{O}_{2}$ ) at $42{ }^{\circ} \mathrm{C}$ for $24 \mathrm{~h}$. When needed, appropriate antibiotics were supplemented at the following concentrations: kanamycin $(50 \mu \mathrm{g}$ $\left.\mathrm{ml}^{-1}\right)$ or chloramphenicol $\left(30 \mu \mathrm{g} \mathrm{ml}^{-1}\right)$. For growth experiments, bacteria were grown at $42{ }^{\circ} \mathrm{C}$ in Mueller-Hinton (MH) broth under microaerophilic conditions in the presence or absence of $10 \mathrm{mM}$ formic acid. All growth experiments were done in triplicate. E. coli strain $\mathrm{DH} 5 \alpha$, which was used for cloning experiments, was grown on Luria-Bertani (LB) agar or in LB broth at $37{ }^{\circ} \mathrm{C}$. When necessary, the medium was supplemented with ampicillin $\left(100 \mu \mathrm{g} \mathrm{ml}^{-1}\right)$.

Generation of competent cells and electroporation. A $10 \mathrm{ml}$ volume of LB broth was inoculated with a single E. coli $\mathrm{DH} 5 \alpha$ colony and incubated overnight at $37{ }^{\circ} \mathrm{C}$ under shaking. Three millilitres of the overnight culture was grown in $500 \mathrm{ml} \mathrm{LB}$ broth at $37{ }^{\circ} \mathrm{C}$ to $\mathrm{OD}_{600}$ 0.35-0.45. The culture was placed on ice for $10 \mathrm{~min}$ and centrifuged for $15 \mathrm{~min}$ at $4000 \mathrm{~g}$ at $4{ }^{\circ} \mathrm{C}$. The pellet was gently resuspended in $50 \mathrm{ml}$ cold water and again centrifuged for $15 \mathrm{~min}$ at $4000 \mathrm{~g}$. This step was repeated three times. Then the pellet was resuspended in $15 \mathrm{ml} 10 \%(\mathrm{v} / \mathrm{v})$ glycerol and centrifuged again. Finally, the cell pellet was carefully dissolved in a final volume of $1 \mathrm{ml}$
$10 \%(\mathrm{v} / \mathrm{v})$ glycerol and $100 \mu \mathrm{l}$ aliquots were used for each electroporation. For the preparation of competent cells of C. jejuni, cells were collected from Columbia blood agar plates and centrifuged at $5000 \mathrm{~g}$ at $4{ }^{\circ} \mathrm{C}$ for $10 \mathrm{~min}$. The cells were resuspended in $1 \mathrm{ml}$ icecold wash buffer containing $272 \mathrm{mM}$ sucrose and $15 \%(\mathrm{v} / \mathrm{v})$ glycerol at $4{ }^{\circ} \mathrm{C}$. This step was repeated three times. Finally, the pellet was resuspended in $400 \mu \mathrm{l}$ washing buffer and $100 \mu \mathrm{l}$ aliquots were used for each transformation.

After the addition of $0.5-3 \mu \mathrm{g}$ plasmid DNA, the mixture was transferred into an ice-cold electroporation cuvette and the cuvette was incubated on ice for $30 \mathrm{~min}$. Electroporation was performed at $2.5 \mathrm{kV}, 25 \mu \mathrm{F}$ and $200 \Omega$ using a BTX Electro Cell Manipulator. Then, $500 \mu \mathrm{l}$ SOC medium was added to the cuvette and the suspension was transferred onto a non-selective Columbia blood agar plate and incubated overnight at $37{ }^{\circ} \mathrm{C}$ under microaerophilic conditions. Finally, the cells were transferred onto a selective plate and incubated at $42{ }^{\circ} \mathrm{C}$ under microaerophilic conditions for a further $2-3$ days.

Construction of the transposon library. Campylobacter coli gene aphA-3 can be used as an antibiotic marker in C. jejuni, in which it confers resistance to kanamycin. In an earlier study, aphA-3 was cloned into plasmid pSB1699 (Colegio et al., 2001). We digested pSB1699 with BamHI, which resulted in the release of the aphA-3 insert. After purification of the insert from the agarose gel, we ligated it into the BamHI-digested pBluescript SKII vector, which resulted in plasmid pBSK-Kan. This plasmid was used as a template for PCR amplification of $1.8 \mathrm{~kb}$ DNA fragments that consisted of the $a p h A-3$ gene flanked by $K p n I$ restriction sites. These amplified fragments were then further subcloned into the KpnI-digested and dephosphorylated transposon construction vector EZ::TN pMOD (Epicentre Biotechnologies), which resulted in EZ::TN pMODKan. The Tn5 transposon named EZ: TN Kan was finally released from pMODKan by digestion with PvuII.

The transposition reaction was carried out by mixing $100 \mathrm{ng}$ gelpurified EZ::TN Kan transposon DNA, $2 \mu \mathrm{l} \mathrm{EZ::TN} \mathrm{transposase}$ (Epicentre Biotechnologies) and $2 \mu 100 \%(\mathrm{v} / \mathrm{v})$ glycerol, followed by incubation for $30 \mathrm{~min}$ at room temperature. To generate random insertional mutants of $C$. jejuni, the clinical isolate B2 was electroporated with $100 \mathrm{ng}$ of the tranposon and the bacteria were incubated for $5 \mathrm{~h}$ at $37{ }^{\circ} \mathrm{C}$ under microaerophilic conditions. The bacteria were subsequently harvested and transformants selected on Columbia blood agar supplemented with $5 \%$ sheep blood and kanamycin, by incubating at $42{ }^{\circ} \mathrm{C}$ under microaerophilic conditions. The same procedure was repeated several times, which produced a library of 660 individual mutants by using eight different signature tags. $\mathrm{MH}$ broth supplemented with $15 \%(\mathrm{v} / \mathrm{v})$ glycerol was used to store the mutants at $-80{ }^{\circ} \mathrm{C}$. Random insertion of the transposons was verified for a fraction of 19 mutants. After preparation of genomic DNA and digestion with $B s p \mathrm{HI}$, a Southern blot analysis was carried out with a digoxigenin-11-UTP-probed kanamycin-resistance gene under conditions described previously (Dasti et al., 2007).

Cultivation of cells. Human colon carcinoma Caco-2 cells were maintained in Dulbecco's minimal essential medium (DMEM) supplemented with $10 \%$ fetal calf serum, $1 \times$ non-essential amino acids, $100 \mathrm{U}$ penicillin $\mathrm{ml}^{-1}$ and $100 \mu \mathrm{g}$ streptomycin $\mathrm{ml}^{-1}$. The cells were routinely cultured in a humidified atmosphere of $95 \%$ air and $5 \% \mathrm{CO}_{2}$ at $37{ }^{\circ} \mathrm{C}$.

Gentamicin protection assays. Bacterial invasion of host cells was initially described by Everest et al. (1992). Prior to the assay, $2 \times 10^{6}$ Caco-2 cells were seeded to each well of a six-well plate. Then, cells were washed with PBS and inoculated with $400 \mu \mathrm{l} C$. jejuni suspension. To ensure that the number of bacteria was identical in every assay performed, the bacterial solution was adjusted to $\mathrm{OD}_{600}$ 0.5 , which represented an m.o.i. of 100 . At $2 \mathrm{~h}$ post-infection, the 
bacterial suspension was removed and the cells were washed three times with $1 \times$ PBS before further incubation with culture medium containing $100 \mu \mathrm{g}$ gentamicin $\mathrm{ml}^{-1}$. Afterwards, cells were lysed with $1 \%$ Triton X-100 for $10 \mathrm{~min}$ to release intracellular bacteria. The number of viable bacteria was determined by plating serial dilutions of the lysate on Columbia blood agar and counting the number of bacteria grown after incubation for $48 \mathrm{~h}$ at $42{ }^{\circ} \mathrm{C}$ under microaerophilic conditions. Means and SDs of the number of colonies counted for the mutant and complemented strains are indicated.

Altogether 660 clones of the generated C. jejuni strain B2 transposon library were screened individually by performing gentamicin protection assays in which every clone was tested in triplicate. Clones that were shown to be less invasive were further investigated in 12 independent experiments to confirm their phenotype of reduced invasiveness.

Motility assay. To test for bacterial motility, $1 \mu \mathrm{l}$ of an overnight culture adjusted to $\mathrm{OD}_{600} 0.025$ was stabbed into the centre of a $0.4 \% \mathrm{MH}$ agar plate with the help of a suitable normalized inoculation loop. Plates were incubated at $42{ }^{\circ} \mathrm{C}$ under microaerophilic conditions for $36 \mathrm{~h}$. The low concentration of the agar allowed the bacteria to swarm, forming a visible halo within the agar. For judgement of motility the expansion of the haloes was examined by measuring the respective diameter. Each experiment was performed seven times.

Chemotaxis assays. The chemotaxis assay was performed as described by Vegge et al. (2009) with the following modifications. Bacteria were grown on Columbia blood agar plates overnight at $42{ }^{\circ} \mathrm{C}$ under microaerophilic conditions. They were then suspended in PBS ( $\mathrm{pH} 7.0$ ), adjusted spectrophotometrically to $\mathrm{OD}_{600} 1$ and mixed (1:1) with tempered soft agar ( $0.8 \%$, Becton Dickinson). Afterwards, $12 \mathrm{ml}$ of the bacterial soft agar suspension was poured into a Petri dish and $6 \mathrm{~mm}$ filter discs (Oxoid) soaked with $20 \mu \mathrm{l}$ of the test chemical were placed on the solidified agar $(0.1 \mathrm{M}$ and pH 7.0; all chemicals were obtained from Sigma-Aldrich). Zones of bacterial attraction or repulsion were measured after $4 \mathrm{~h}$ incubation at $42{ }^{\circ} \mathrm{C}$ under microaerophilic conditions. The chemotaxis assays were carried out four times.

Isolation of nucleic acids. Genomic DNA of C. jejuni was isolated using the QIAamp DNA Mini kit (Qiagen) according to the manufacturer's instructions. Bacterial RNA was extracted from $C$. jejuni cells with the RiboPure Bacteria kit (Ambion) following the recommendations of the manufacturer's protocol. After RNA isolation, DNA contamination was removed by DNase I treatment (Ambion) and incubation at $37{ }^{\circ} \mathrm{C}$ for $30 \mathrm{~min}$, followed by denaturation of the enzyme for $15 \mathrm{~min}$ at $75{ }^{\circ} \mathrm{C}$. After DNase I treatment, PCR assays were performed to make sure that the RNA was free of remaining traces of DNA. DNA and RNA concentrations were determined with a NanoDrop ND 1000 spectrophotometer (Thermo Scientific).

Sequencing of genomic DNA of $\boldsymbol{C}$. jejuni. Ten micrograms of genomic DNA was sequenced directly by Seqlab (Göttingen, Germany) using 10 pmol of the KanF primer listed in Table 1.

RT-PCR analysis. Conventional RT-PCR assays were carried out with the OneStep RT-PCR kit (Qiagen) as instructed by the manufacturer. After reverse transcription of $10 \mathrm{ng}$ for $30 \mathrm{~min}$ at $50{ }^{\circ} \mathrm{C}, 35$ cycles of amplification were carried out according to the following protocol: cDNA denaturation at $94{ }^{\circ} \mathrm{C}$ for $30 \mathrm{~s}$, primer annealing at $55{ }^{\circ} \mathrm{C}$ for $30 \mathrm{~s}$ and extension at $72{ }^{\circ} \mathrm{C}$ for $1 \mathrm{~min}$, with a final incubation at $72{ }^{\circ} \mathrm{C}$ for $10 \mathrm{~min}$. PCR products were analysed on a $1 \%$ agarose gel containing ethidium bromide at a final concentration of $1 \mu \mathrm{g} \mathrm{ml}^{-1}$. The primers used for RT-PCR are listed in Table 1.

PCR and RT-PCR assays for nucleic acid alignment. To compare the DNA and RNA sequence between cj0952c and downstream cj0951c, PCR and RT-PCR assays were carried out using the primers Cj0952c-51cF and Cj0952c-51cR (Table 1) for the amplification of a $460 \mathrm{bp}$ fragment encompassing the $3^{\prime}$ domain of $c j 0952 c$, the intergenic region and the $5^{\prime}$ domain of cj0951c. PCR was carried out using a TRIO-Thermoblock (Biometra) with genomic DNA of $C$. jejuni strain $\mathrm{B} 2$ as template. Each $50 \mu \mathrm{l}$ of PCR mixture contained 40 ng genomic DNA, $10 \mathrm{mM}$ Tris/ $\mathrm{HCl}, \mathrm{pH} 8.3,50 \mathrm{mM} \mathrm{KCl}, 1.5 \mathrm{mM}$ $\mathrm{MgCl}_{2}$, all four dNTPs $(0.2 \mathrm{mM}$ each) and $2.5 \mathrm{U}$ Taq DNA polymerase (Roche). Initial incubation at $95{ }^{\circ} \mathrm{C}$ for $1 \mathrm{~min}$ was followed by 40 cycles at $95{ }^{\circ} \mathrm{C}$ for $30 \mathrm{~s}, 55{ }^{\circ} \mathrm{C}$ for $30 \mathrm{~s}$ and $72{ }^{\circ} \mathrm{C}$ for $1 \mathrm{~min}$, with a final incubation at $72{ }^{\circ} \mathrm{C}$ for $10 \mathrm{~min}$. The RT-PCR assay was carried out with the OneStep RT-PCR kit as instructed by the manufacturer in a TRIO-Thermoblock. Briefly, 80 ng total RNA was reverse-transcribed for $30 \mathrm{~min}$ at $50{ }^{\circ} \mathrm{C}$. Following cDNA synthesis, HotStarTaq DNA polymerase (Qiagen) was initially activated for $15 \mathrm{~min}$ at $94{ }^{\circ} \mathrm{C}$. Then, 40 cycles of amplification were carried out according to the following protocol: cDNA denaturation at $94{ }^{\circ} \mathrm{C}$ for $30 \mathrm{~s}$, primer annealing at $55^{\circ} \mathrm{C}$ for $30 \mathrm{~s}$ and extension at $72{ }^{\circ} \mathrm{C}$ for $1 \mathrm{~min}$, with a final incubation at $72{ }^{\circ} \mathrm{C}$ for $10 \mathrm{~min}$. After direct sequencing of the PCR amplicons by Seqlab with the primers used for amplification, derived sequences were compared by performing BLASTN analysis.

Real-time RT-PCR analysis. Semi-quantitative real-time RT-PCR analysis was carried out with a LightCycler 1.5 instrument (Roche) and the QuantiFast SYBR Green RT-PCR kit (Qiagen). Fifty nanograms of isolated RNA from $C$. jejuni B2 wild-type strain and from B2 $\Delta c j 0952 c$ complemented with $c j 0952 c$ and $c j 0952 c-c j 0951 c$ was used to compare the transcription levels of $c j 0952 c$. Amplicons of the $23 \mathrm{~S}$ rRNA gene, which is transcribed constitutively, were used to adjust the cj0952c RT-PCR based on the crossing points obtained for this gene. $23 \mathrm{~S}$ rRNA RT-PCR was performed as follows: after initial reverse transcription for $20 \mathrm{~min}$ at $50{ }^{\circ} \mathrm{C}$ and denaturation for $5 \mathrm{~min}$ at $95{ }^{\circ} \mathrm{C}, 40$ cycles of denaturation $\left(95^{\circ} \mathrm{C}, 10 \mathrm{~s}\right)$, annealing $\left(50{ }^{\circ} \mathrm{C}\right.$, $10 \mathrm{~s})$ and elongation $\left(72{ }^{\circ} \mathrm{C}, 5 \mathrm{~s}\right)$ were carried out. After adjustment of the RNA samples with regard to the crossing points determined for the 23S rRNA gene, real-time RT-PCR assays for the amplification of $c j 0952 c$ were run. The elongation time for the amplification of $c j 0952 c$ was shortened to ensure that only wild-type $c j 0952 c$, and not cj0952c harbouring the transposon, was amplified. Reverse transcription for $20 \mathrm{~min}$ at $50{ }^{\circ} \mathrm{C}$ and denaturation for $5 \mathrm{~min}$ at $95{ }^{\circ} \mathrm{C}$ were followed by 40 cycles of denaturation $\left(95^{\circ} \mathrm{C}, 10 \mathrm{~s}\right)$, annealing $\left(55^{\circ} \mathrm{C}, 10 \mathrm{~s}\right)$ and elongation $\left(72{ }^{\circ} \mathrm{C}, 10 \mathrm{~s}\right)$. The primers used for both assays are shown in Table 1. Every assay was run in duplicate. The presence of the signal corresponding to wild-type $c j 0952 c$ but not to transposoninterrupted $c j 0952 c$ was ensured by melting-curve analysis and agarose gel electrophoresis (results not shown). Semi-quantitative measurement of transcription levels was calculated as follows:

$x=2^{\Delta \mathrm{Cp}}$

where $x$ represents the factor of altered transcription, and $\Delta \mathrm{Cp}$ represents the difference in crossing points $(\mathrm{Cp} 1-\mathrm{Cp} 2)$ of two samples to be compared.

Cloning of $\boldsymbol{C}$. jejuni genes into expression vector pRRC. The $C$. jejuni genes cj0952c and cj0951c alone, cj0952c-cj0951c, cj0952c$c j 0951 c-c j 0950 c$ and gene $c j 0951 c$ from $C$. jejuni strain 81-176 were PCR-amplified and subsequently cloned into vector pRRC using primers harbouring restriction sites for XbaI (Table 1). The Campylobacter vector pRRC contains a $\mathrm{cam}^{\mathrm{R}}$ gene cassette that is flanked by a $16 \mathrm{~S}$ rRNA sequence and sequences for tRNAs for alanine and isoleucine. A single XbaI site is located immediately downstream 
Table 1. Oligonucleotide primers used for sequencing, RT-PCR analysis and cloning of C. jejuni genes

$\mathrm{Xba \textrm {I }}$ restriction sites for cloning in pRRC are underlined, the start codon of $c j 0952 c$ is shown in bold type and the putative SD sequence is shown in italic type.

\begin{tabular}{|c|c|c|}
\hline Gene & Primer & Sequence $\left(5^{\prime}-3^{\prime}\right)$ \\
\hline & Sequencing & \\
\hline \multirow{2}{*}{$a p h A-3$} & KanF & TATCACCTCAAATGGTTCGCT \\
\hline & RT-PCR & \\
\hline \multirow[t]{2}{*}{ cj0951c } & Cj0951F & GAGTTACCAAAGCCCTAGCATC \\
\hline & Cj0951R & CATGGTCAATCAAGCAGG \\
\hline \multirow[t]{2}{*}{$c j 0952 c$} & Cj0952F & GCCACTTCCTTGAGC \\
\hline & Cj0952R & CGCCCTTTGATTGCAGATG \\
\hline \multirow[t]{2}{*}{$c j 0953 c$} & Cj0953F & GGCTGAAATGATTACTTCTAC \\
\hline & Cj0953R & CTAACCGATATTAACGCAGC \\
\hline \multirow[t]{3}{*}{$23 S$} & Cj23SF & GTTCGCCATTTAAAGCGGTA \\
\hline & Cj23SR & TGCTCTTGGCAGAACAACAG \\
\hline & Alignment & \\
\hline \multirow{3}{*}{$c j 0952 c-51 c$} & Cj0952c-51cF & GCCAATTCATTTTCATTTTGAGA \\
\hline & Cj0952c-51cR & TTTTAATGCTTAAAGTTGTTGTTGG \\
\hline & Cloning in pRRC & \\
\hline \multirow[t]{2}{*}{ cj0952c } & Cj0952cF & GCTCTAGATAGGAAACTATGATGTTTAAAACTA \\
\hline & $\mathrm{Cj0952cR}$ & GCTCTAGAAATTAATTGATATATCCACA \\
\hline \multirow[t]{2}{*}{ cj0951c } & $\mathrm{Cj} 0951 \mathrm{cF}$ & NNNTCTAGAATTTTATCGAAAAAACTCATAAGG \\
\hline & Cj0951cR & NNNTCTAGATTAAATTTGAAATTGGTTAAGTTCGC \\
\hline cj0952cl & $\mathrm{Cj0952cF}$ & GCTCTAGATAGGAAACTATGATGTTTAAAACTA \\
\hline cj0951c & Cj0951cR & NNNTCTAGACCTTTAAATTTGAAATTGGTTAAGTTCGC \\
\hline cj0952cl & $\mathrm{Cj} 0952 \mathrm{cF}$ & GCTCTAGATAGGAAACTATGATGTTTAAAACTA \\
\hline cj0951cHis & Cj0951His & GGCTCTAGATTAGTGATGGTGATGGTGATGAATTTGAAATTGGTTAAGTTCGC \\
\hline cj0952cl & $\mathrm{Cj} 0952 \mathrm{~F}$ & GCTCTAGATAGGAAACTATGATGTTTAAAACTA \\
\hline cj0950c & Cj0950R & NNNTCTAGAGGGCATTATTTAAAAAAGATTTTGATTTC \\
\hline
\end{tabular}

of the $\mathrm{cam}^{\mathrm{R}}$ gene cassette, and allows the expression of XbaI-cloned genes under the control of the constitutively expressed $c a m^{\mathrm{R}}$ gene promoter. After introduction of the plasmid into competent cells of C. jejuni, the $\mathrm{cam}^{\mathrm{R}}$ gene cassette together with the XbaI-cloned gene was integrated into one of the $16 \mathrm{~S}$ rRNA loci of the recipient cell via highly efficient double recombination (Karlyshev \& Wren, 2005).

PCR was carried out in a TRIO-Thermocycler (Biometra) with $10 \mathrm{ng}$ genomic DNA of C. jejuni strain B2 as template. Each $50 \mu$ of PCR mixture contained $0.02 \mathrm{U}$ KOD Hot Start DNA Polymerase (Novagen), dNTPs (0.2 mM each), $1.5 \mathrm{mM} \mathrm{MgSO} 4,10$ pmol of each primer and $1 \times \mathrm{KOD}$ Hot Start buffer. Initial incubation at $95{ }^{\circ} \mathrm{C}$ for $3 \mathrm{~min}$ was followed by 40 cycles at $95{ }^{\circ} \mathrm{C}$ for $30 \mathrm{~s}, 55^{\circ} \mathrm{C}$ for $30 \mathrm{~s}$ and $72{ }^{\circ} \mathrm{C}$ for $1 \mathrm{~min}$, with a final incubation at $72{ }^{\circ} \mathrm{C}$ for $5 \mathrm{~min}$. The PCR products were purified with the QIAquick PCR Purification kit (Qiagen) and digested with XbaI (New England Biolabs). Plasmid pRRC was also XbaI-restricted and dephosphorylated using Antarctic Phosphatase (New England Biolabs). The restricted PCR fragments were ligated into pRRC using the Quick Ligase kit (New England Biolabs) according to the manufacturer's instructions. After transformation of competent C. jejuni $\mathrm{B} 2 \Delta c j 0952 c$ cells, the complementation constructs were verified by PCR analysis for the detection of the respective genes.

Purification of His-tagged proteins and immunoblot analysis. For the detection of His-tagged proteins, C. jejuni cells were grown in $500 \mathrm{ml} \mathrm{MH}$ broth at $42{ }^{\circ} \mathrm{C}$ for $36 \mathrm{~h}$ under microaerophilic conditions $\left(85 \% \mathrm{~N}_{2}, 10 \% \mathrm{CO}_{2}, 5 \% \mathrm{O}_{2}\right)$. All the following steps were carried out according to the protocol from The QIAexpressionist with the solutions recommended for purification of $6 \times$ His-tagged proteins under denaturing conditions (Qiagen). Briefly, after lysis of the cells, $1 \mathrm{ml} 50 \%$ Ni-NTA slurry was added and after shaking for $1 \mathrm{~h}$, the lysate-resin mixture was loaded into an empty column. After removal of the flowthrough, the Ni-NTA slurry was washed twice with buffer C followed by elution of the His-tagged proteins with buffers D and E. Aliquots of $10 \mu \mathrm{l}$ were separated on $15 \%$ SDS-PAGE and transferred to a PVDF membrane (GE Healthcare) using a Semi-Dry Transfer system (Sartorius). The membrane was blocked for $1 \mathrm{~h}$ with $5 \%$ milk powder in PBS containing $0.05 \%$ Tween 20 and incubated in blocking solution. After incubation of the membrane with a $1: 3000$ diluted monoclonal mouse anti-His primary antibody (Qiagen) overnight at $4{ }^{\circ} \mathrm{C}$, the immune complexes were labelled with a 1:3000-diluted horseradish peroxidase-conjugated anti-mouse secondary antibody (Dianova) for $1 \mathrm{~h}$ at room temperature and visualized by ECL chemiluminescence.

Statistical analysis. Significant differences between mean values were calculated by Student's $t$ test using the STATISTICA software (StatSoft). $P$ values of less than 0.01 were considered to be significant.

\section{RESULTS}

\section{Invasion of Caco-2 cells by C. jejuni isolate B2 and $C$. jejuni strains NCTC 11168 and 81-176}

To initially determine the capability of $C$. jejuni strain $\mathrm{B} 2$ to invade Caco- 2 cells we compared this isolate with the wellcharacterized C. jejuni strains NCTC 11168 and 81-176. 
Performing gentamicin protection assays, we could clearly show that isolate B2 is more invasive than both of these reference strains. While the mean number of colonies recovered from isolate B2 was $1.2 \times 10^{6}$ c.f.u.ml ${ }^{-1}, 0.8 \times 10^{5}$ and $4.1 \times 10^{5}$ c.f.u. $\mathrm{ml}^{-1}$ were obtained for reference strains NCTC 11168 and 81-176, respectively. The results are shown in Fig. 1(a).

\section{Identification of cj0952c}

To identify genes which contribute to the ability of C. jejuni to invade Caco-2 cells, we screened individually altogether 660 clones of a transposon-based mutant library of $C$. jejuni strain B2 by performing gentamicin protection assays. Thereby, seven clones with a strongly decreased invasiveness could be detected. The transposon insertion site of the respective $C$. jejuni clones was mapped by direct sequencing of genomic DNA using a primer that binds directly to the $5^{\prime}$ region of the kanamycin-resistance cassette and therefore allows the identification of the affected C. jejuni genes. To confirm the decreased invasiveness, gentamicin protection assays with the respective clones and parental strain $\mathrm{B} 2$ were repeated five times. Thereby, the mean number of colonies recovered from B2 was $1.1 \times 10^{6}$ c.f.u. $\mathrm{ml}^{-1}$. The mean c.f.u. $\mathrm{ml}^{-1}$ values obtained for the respective mutants were as follows: cj0005c, $2.8 \times 10^{5}$ c.f.u. $\mathrm{ml}^{-1}$; cj0078c, $3.4 \times 10^{5}$ c.f.u. $\mathrm{ml}^{-1}$; cj0093, $4.1 \times 10^{5}$ c.f.u. $\mathrm{ml}^{-1}$; cj0268c, $3.3 \times 10^{5}$ c.f.u. $\mathrm{ml}^{-1}$; cj0721c, $3.2 \times 10^{5}$ c.f.u. $\mathrm{ml}^{-1}$; cj1439c, $3 \times 10^{5}$ c.f.u. $\mathrm{ml}^{-1}$; and $c j 0952 c, 2.3 \times 10^{5}$ c.f.u. $\mathrm{ml}^{-1}$. Since the $P$ value for

(a)

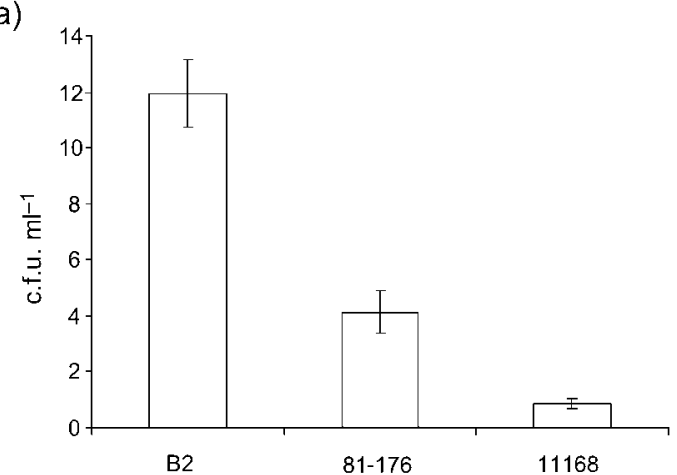

(b)

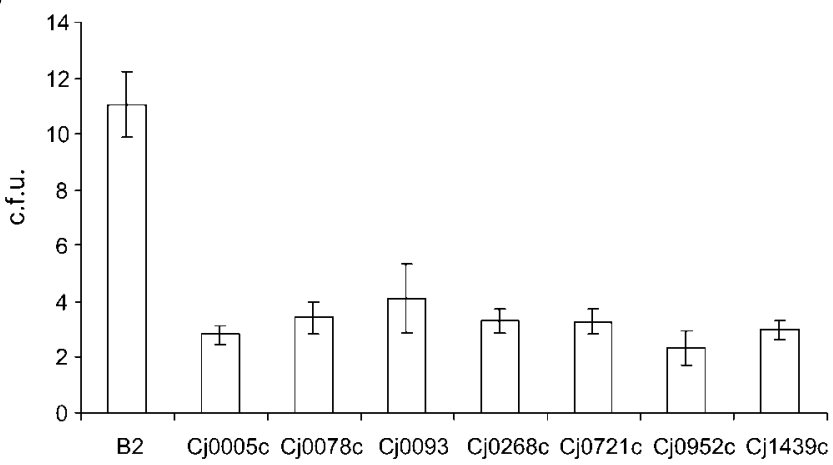

(c)

\begin{tabular}{ll}
\hline Gene & \multicolumn{1}{c}{ Putative function } \\
\hline$c j 0005 c$ & Sulphite $:$ cytochrome c oxidoreductase \\
$c j 0078 c$ & CdtB, cytolethal distending toxin \\
$c j 0093$ & Periplasmic protein \\
$c j 0268 c$ & Transmembrane protein \\
$c j 0721 c$ & Integral membrane protein \\
$c j 0952 c$ & N terminus of a chemoreceptor \\
$c j 1439 \mathrm{C}$ & UPD-galactopyranose mutase \\
\hline
\end{tabular}

Fig. 1. (a) Comparison of the capability of the C. jejuni strains B2, NCTC 11168 and 81176 to invade Caco- 2 cells. The mean values of colonies recovered from NCTC 11168 and 81-176 were $0.8 \times 10^{5}$ and $4.1 \times 10^{5}$ c.f.u. $\mathrm{ml}^{-1}$. In contrast, the number of colonies obtained from strain B2 was $1.2 \times 10^{6}$ c.f.u. $\mathrm{ml}^{-1}$, demonstrating the increased infectivity of this clinical isolate. (b) A general view of genes detected in our study that mediate reduced invasiveness of C. jejuni strain B2. (c) Description of the detected genes concerning function and localization as far as known. See text for details. 
every mutant tested was less than 0.001, the reduced invasion capacities were judged to be significant. An overview of the genes mapped is given in Fig. 1(b, c). One of the clones with strongly decreased invasion capacity exhibited insertion of the transposon in gene cj0952c at position 106 according to C. jejuni strain NCTC 11168, and we chose this mutant as the object of further investigations.

\section{Insertion of the transposon in cj0952c does not affect transcription of either the upstream gene cj0953c or the downstream gene cj0951c}

To find out whether the insertion of the transposon into cj0952c has any impact on the transcription of the adjacent genes, we carried out rRT-PCR assays with the wild-type strain B2 and the mutant B2 $\Delta c j 0952 c$. Since the non-coding sequence between $c j 0953 c$ and $c j 0952 c$ has a length of $96 \mathrm{bp}$, and the distance between $c j 0952 \mathrm{c}$ and $c j 0951 \mathrm{c}$ is $263 \mathrm{bp}$ in the clinical isolate B2, transcription of these three genes in a polycistronic manner cannot be excluded. Accordingly, insertion of a transposon in one gene may interrupt transcription, particularly of genes located downstream. As shown in Fig. 2, transcription of cj0952c including the transposon, but also of $c j 0953 c$ and $c j 0951 c$, could clearly be detected by RT-PCR in the mutant $\mathrm{B} 2 \Delta c j 0952 c$, indicating that the invasion-deficient phenotype is exclusively mediated by the insertion of the transposon into cj0952c.

\section{Functional complementation of $C$. jejuni B2 $\Delta c j 0952 c$}

In order to restore the invasiveness of the $\mathrm{B} 2 \Delta c j 0952 c$ mutant, $c j 0952 c$ and $c j 0951 c$ alone and $c j 0952 c$ together with $c j 0951 c$ were cloned into vector $\mathrm{pRRC}$ and introduced into mutant B2 $\Delta c j 0952 c$ by electroporation. Performing gentamicin protection assays on Caco- 2 cells, the number of C. jejuni colonies recovered demonstrated that $c j 0952 c-$ cj0951c, but not $c j 0952 c$ or cj0951c alone, altered the mutant phenotype to that of wild-type B2. The results of five independent experiments are shown in Fig. 3(a, b). In contrast to wild-type strain B2, transcription of $c j 0952 c$ in the complemented mutants is under control of a $\mathrm{cam}^{\mathrm{R}}$ promoter. To compare the transcription levels of $c j 0952 c$, we carried out real-time RT-PCR analysis. After adjustment of the RNA samples according to the crossing points of the 23S rRNA transcripts, we investigated the cj0952c transcription level of the wild-type strain and of the mutant B2 $\Delta c j 0932 c$ complemented with $c j 0952 c$ alone and with cj0952c-cj0951c. Surprisingly, while the mean crossing points of both complemented mutants were 20.49 (cj0952c) and $20.18(c j 0952 c-c j 0951 c)$, the mean crossing point for the detection of $c j 0952 c$ with RNA from the wildtype strain was 32.44 , demonstrating a more than 4000fold upregulation of $c j 0952 c$ in the complemented mutants compared with the wild-type strain. Accordingly, lower transcription of $c j 0952 c$ could be excluded as a reason for the incomplete invasion phenotype.

\section{(a)}

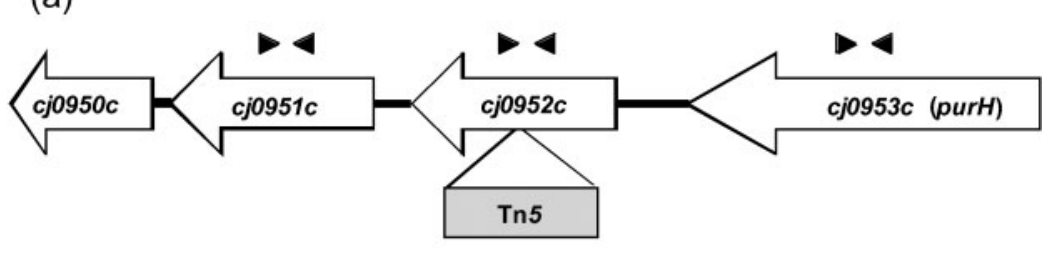

(b)

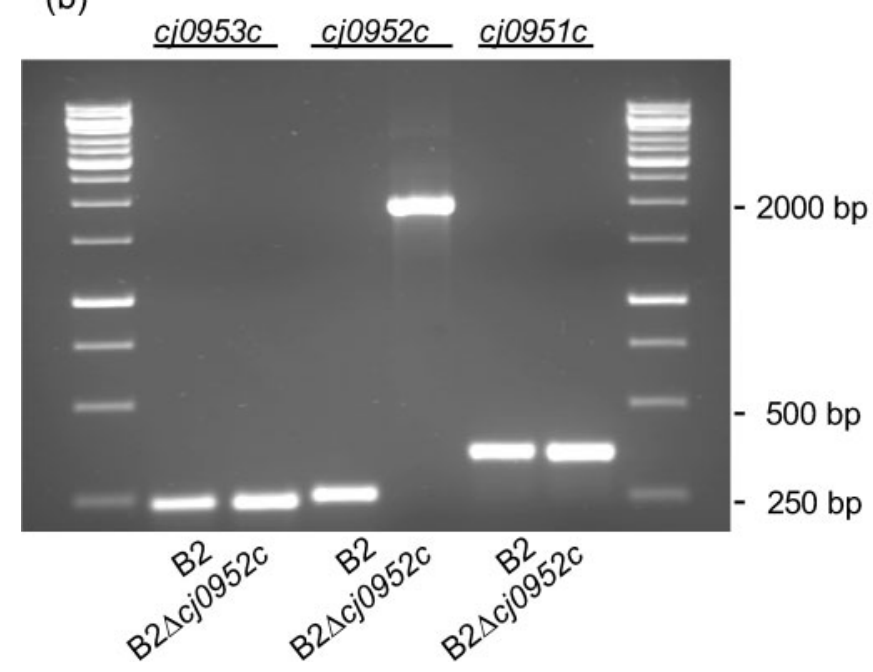

Fig. 2. (a) Genome arrangement of cj0953c, cj0952c, cj0951c and cj0950c in the clinical C. jejuni isolate B2 (GenBank accession no. GU799572). The direction of the arrows denotes the direction of transcription. Primers are shown as arrowheads. The insertion site of the transposon is indicated. (b) RT-PCR analysis for the investigation of $C$. jejuni genes cj0953c, cj0952c and cj0951c in the wildtype B2 strain and the mutant strain B2Acj0952c. The PCR amplicons show the expected size for cj0953c (225 bp), cj0952c wild-type (267 bp), cj0952c with transposon insert (2091 bp) and cj0951c (356 bp). PCR analysis of the RNA templates under investigation yielded no amplicons, demonstrating that DNA contamination was completely removed (not shown). Primers used for RTPCR assays are listed in Table 1. 


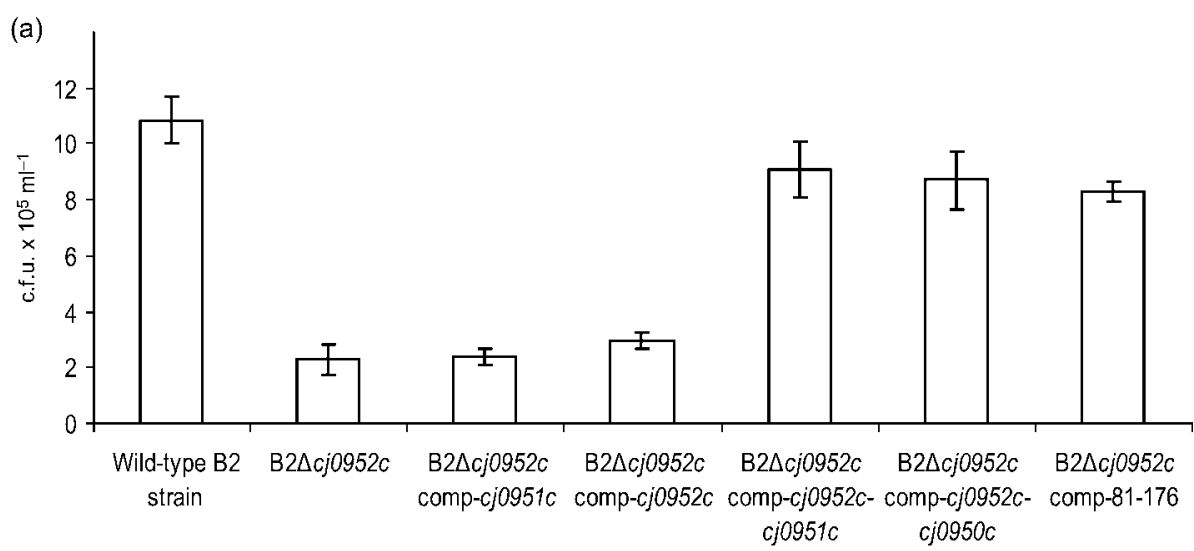

(b)

\begin{tabular}{|c|c|c|}
\hline Strain & c.f.u. $\mathrm{ml}^{-1}\left(\times 10^{5}\right)$ & $\operatorname{SD}\left(\times 10^{5}\right)$ \\
\hline Wild-type B2 & 10.86 & \pm 0.86 \\
\hline $\mathrm{B} 2 \wedge c j 0952 c$ & 2.30 & \pm 0.53 \\
\hline $\mathrm{B} 2 \Delta c j 0952 c$-comp-cj0952c & 2.98 & \pm 0.28 \\
\hline $\mathrm{B} 2 \Delta c j 0952 c$-comp-cj0951c & 2.38 & \pm 0.26 \\
\hline $\mathrm{B} 2 \Delta c j 0952 c$-comp-cj0952c-cj0951c & 9.08 & \pm 1.00 \\
\hline $\mathrm{B} 2 \Delta c j 0952 c-\mathrm{comp}-c j 0952 c-c j 0950 c$ & 8.72 & \pm 1.04 \\
\hline B2 $\Delta c j 0952 c$-comp-81-176 & 8.30 & \pm 0.37 \\
\hline
\end{tabular}

Fig. 3. (a) Infection of Caco-2 cells. Restoration of invasiveness to the level of the C. jejuni wild-type B2 strain was successful by complementation of B2 $\Delta c j 0952 c$ with $c j 0952 c-c j 0951 c$ but not with cj0952c or cj0951c alone. The additional expression

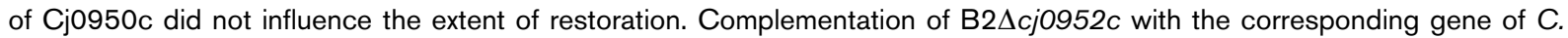
jejuni strain 81-176 (B2 $\Delta$ cj0952c-comp-81-176) gave the same extent of restoration as complementation of the mutant with cj0952c-cj0951c from the parental isolate B2. (b) Mean numbers of colonies recovered after five independent experiments; SDs are indicated. While the reduced invasiveness of the mutant B2 $\Delta c j 0952 \mathrm{c}$ and the mutants complemented with $c j 0952 \mathrm{c}$ or cj0951c was significant compared with the parental strain B2 $(P<0.001)$, the variation in recovered colony numbers after invasion assays with mutants complemented with cj0952c-cj0951c, cj0952c-cj0950c or 81-176 was not significant $(P>0.001)$.

In contrast to the clinical isolate $\mathrm{B} 2$ and $C$. jejuni strain NCTC 11168, in which the putative chemoreceptor Cj0952c-Cj0951c is composed of two genes, the corresponding protein in $C$. jejuni strain $81-176$ is encoded by only one gene that covers the transmembrane domain, the HAMP domain as well as the MCP domain. To investigate whether the single gene of $C$. jejuni $81-176$ is able to complement B2 $\Delta c j 0952 c$, we cloned it into pRRC and introduced the recombinant plasmid into the mutant. Subsequent invasion assays with Caco-2 cells restored the invasiveness to wild-type levels, and this is summarized in Fig. 3(a, b). In a further attempt to fully restore the invasiveness of the B2 $\Delta c j 0952 c$ mutant, we cloned a PCR fragment encompassing cj0952c-cj0951c-cj0950c into vector pRRC. The cj0950c gene is located downstream of cj0951c and encodes a putative lipoprotein of unknown function. It contains a predicted signal peptide and shows similarities to heat-shock protein HSLJ of E. coli. Since nothing is known about the role of this gene, a functional correlation of Cj0950c with Cj0952c and Cj0951c could not be excluded. After introduction of the recombinant plasmid carrying all three genes, gentamicin protection assays with Caco- 2 cells were again carried out. In these assays, the recovery rate of colonies compared with the mutant complemented with cj0952c-cj0951c was not significantly different, indicating that Cj0950c does not have a functional relationship with Cj0952 and Cj0951c. The results are summarized in Fig. 3(a,b).

\section{C. jejuni genes cj0952c and cj0951c of the clinical isolate $\mathrm{B2}$ are not translated as one protein}

C. jejuni genes $c j 0952 c$ and $c j 0951 c$ in the clinical isolate B2 as well as in strain NCTC 11168 are thought to be pseudogenes, and a read-through mechanism is postulated to create one functional protein. We carried out PCR and 
RT-PCR with DNA and RNA of the parental strain B2 to answer the question of whether the synthesized RNA is altered during the process of transcription in such a way that, for example, the stop codon of $c j 0952 c$ is replaced to create one ORF for both genes. PCR and RT-PCR assays were performed with the primers $\mathrm{Cj} 0952 \mathrm{c}-51 \mathrm{cF}$ and Cj0952c-51cR (Table 1), which span the $3^{\prime}$ region of $c j 0952 c$, the intergenic region and the $5^{\prime}$ domain of $c j 0951 c$. After sequencing of the PCR and RT-PCR products and subsequent alignment we could not detect any differences between the two amplicons. The stop codon of cj0952c at position 891663 of the genome was not converted and nor could other changes of the RNA sequence be detected, which clearly demonstrated that no read-through mechanism can be postulated based on information obtained from the RNA sequence (data not shown). However, by the detection of an RT-PCR amplicon, we could verify that $c j 0951 c$ and $c j 0952 c$ are cotranscribed on one mRNA. To determine whether Cj0952c and Cj0951c are translated as one protein or separately as single proteins, we cloned both genes together into pRRC. For the detection of the protein expressed in trans, the primer corresponding to the $3^{\prime}$ end of $c j 0951 c$ was expanded by nucleotides representing a $6 \times$ His-tag (Table 1). After introduction of the recombinant plasmid into the mutant $\mathrm{B} 2 \Delta c j 0952 c$, we lysed cells of the complemented mutant and of parental strain B2, which served as a negative control in the following immunoblot experiment. The proteins of both pools were purified on a Ni-NTA matrix, separated by SDS-PAGE, blotted and finally stained using a monoclonal antibody against the His-tag. Thereby, we could clearly detect a band corresponding to approximately $25 \mathrm{kDa}$, which agrees with the predicted protein size of Cj0951c but not with the expected size for Cj0952c-Cj0951c $(58 \mathrm{kDa})$, indicating that no read-through mechanism takes place in $C$. jejuni strain B2 (Fig. 4). We repeated the experiment with other randomly chosen clones obtained after electroporation and

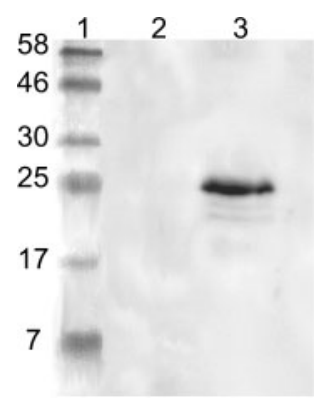

Fig. 4. Immunoblot for the detection of the recombinant protein in C. jejuni. Lanes: 1, molecular mass marker (molecular masses are indicated in $\mathrm{kDa}$ ); 2 , wild-type strain $\mathrm{B} 2 ; 3$, mutant $\mathrm{B} 2 \Delta$ cj0952ccomp-cj0952c-cj0951cHis. The stained molecule exhibits a molecular mass of approximately $25 \mathrm{kDa}$, which is in accordance with the size of Cj0951c. In contrast, a protein assembled from Cj0952c and Cj0951c would have a predicted molecular mass of $58 \mathrm{kDa}$. confirmed the results of the immunoblot analysis. While in most of the clones expression of the recombinant protein could not be detected, two clones showed a band corresponding to $25 \mathrm{kDa}$ after immunostaining. A recombinant protein with a size of approximately $58 \mathrm{kDa}$ was never observed (data not shown).

\section{C. jejuni mutant B2 $\Delta c j 0952 \mathrm{c}$ possesses decreased motility}

Since it is well known that chemotaxis of the pathogen is linked to motility (Hugdahl et al., 1988), we used motility assays to determine whether the B2 $\Delta c j 0952 c$ mutant has an altered phenotype. Thereby, we could ascertain that the motility of the mutant was clearly reduced compared with that of the parental strain B2. While the mean diameter of the motility zone of $\mathrm{B} 2$ was $47.4 \pm 3.13 \mathrm{~mm}$, the mutant was significantly less motile $(30.6 \pm 2.4 \mathrm{~mm}, P<0.0001)$. After complementation of the mutant with $c j 0952 c$ or cj0951c, the motility was not altered $(30.6 \pm 2.9 \mathrm{~mm}$ or $29.4 \pm 2.3 \mathrm{~mm}$, respectively) and remained significantly reduced compared with that of the parental strain $(P<0.0001)$. In contrast, when $c j 0952 c$ together with $c j 0951 c$ was introduced into the mutant, the complementation restored the motility to the wild-type level (46.6 \pm $3.2 \mathrm{~mm}$ ). We were also successful in restoring the parental phenotype after introduction of the corresponding gene from C. jejuni 81-176. The motility level of this revertant was $46.2 \pm 2.8 \mathrm{~mm}$. The results are shown in Fig. 5 .

\section{Cj0952c-Cj0951c alters chemotactic behaviour of C. jejuni in the presence of formic acid}

In order to investigate the function of Cj0952c-Cj0951c, we compared the chemotactic behaviour of the B2 $\Delta c j 0952 c$ mutant with that of the wild-type strain B2. For both, we could detect attraction towards L-asparagine, L-aspartate, L-cysteine, fumarate, L-glutamate, D-lactate, L-(-)-malate, pyruvate, L-serine and succinate. Repulsion was demonstrated from cholic acid, deoxycholic acid, glycocholic acid and taurocholic acid, while no taxis response could be detected from PBS, L-fucose and citrate. No significant differences in the extent of attraction or repulsion between wild-type strain B2 and the mutant B2 $\Delta$ cj0952c could be identified (results not shown).

In contrast, a clear difference could be observed for formic acid. Formic acid has recently been described as chemoattractant for C. jejuni (Vegge et al., 2009). In accordance with the results of Vegge and co-workers, we were able to demonstrate a significant attraction halo around the fomate-soaked paper disc (Fig. 6a). In contrast, a biphasic halo with an inner repulsion zone surrounded by a faint ring was observed for $\mathrm{B} 2 \Delta c j 0952 c$. Complementation of the mutant with $c j 0952 c$ or $c j 0951 c$ alone did not reconstitute the chemotactic behaviour, while complementation with cj0952c-cj0951c or the corresponding gene from C. jejuni 81-176 did reconstitute chemotactic behaviour (Fig. 6a). 
(a)

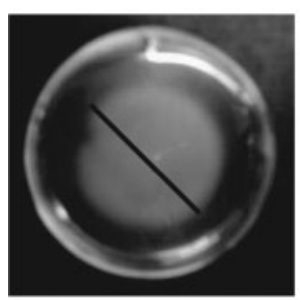

Wild-type B2 strain

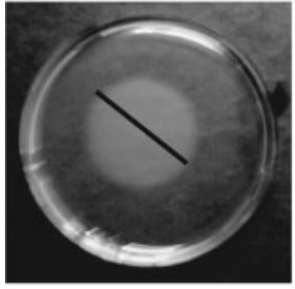

$\mathrm{B} 2 \Delta c j 0952 c$ comp-cj0952c

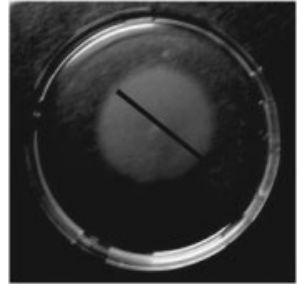

$\mathrm{B} 2 \Delta c j 0952 c$

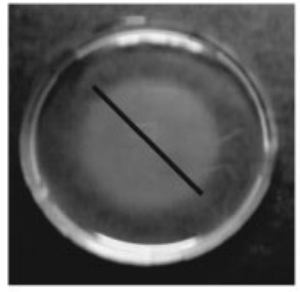

B2 $\Delta$ cj0952c-compcj0952c-cj0951c

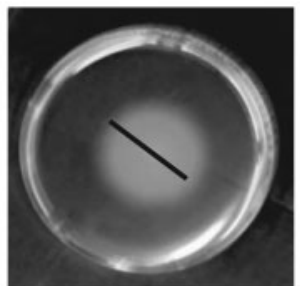

$\mathrm{B} 2 \Delta c j 0952 c-$ comp-cj0951c

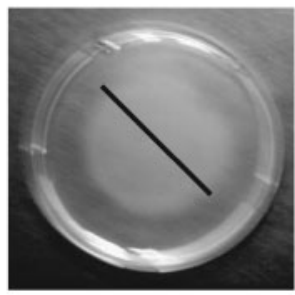

$\mathrm{B} 2 \Delta c j 0952 c$ comp-81-176

(b)

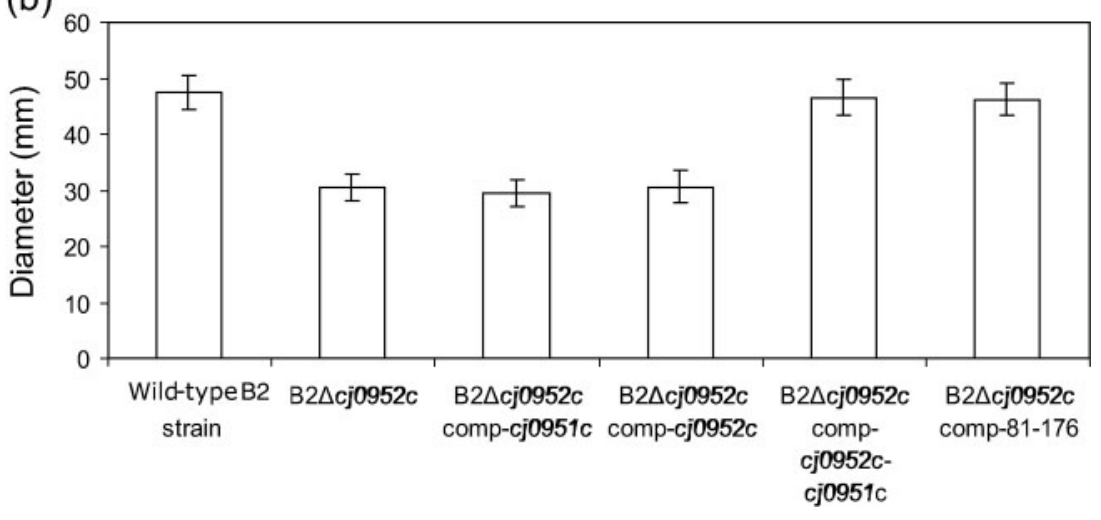

Fig. 5. (a) Motility of wild-type B2 strain, B2 $\Delta c j 0952 c$, B2 $\Delta$ cj0952c-comp-cj0951c, B2 $\Delta$ cj0952c-comp-cj0952c, B2 $\Delta$ cj0952ccomp-cj0952c-cj0951c and the mutant complemented with the corresponding gene from strain 81-176 (B2Acj0952c-comp-81-176). The reduced motility of $\mathrm{B} 2 \Delta \mathrm{cj} 0952 \mathrm{c}$ compared with wild-type strain B2 was not affected by the introduction of cj0952c or cj0951c alone, but was affected by the combination of cj0952c and cj0951c or by the introduction of the corresponding gene of C. jejuni strain 81-176. Diagonal lines show the diameters of the bacterial spread on the agar plates. (b) Diameters of the motility zones of wild-type strain B2, the mutant B2Acj0952c and the complemented mutants. Motility assays were performed on $0.4 \% \mathrm{MH}$ agar plates inoculated with the indicated strains and incubated at $42{ }^{\circ} \mathrm{C}$ under microaerophilic conditions for $36 \mathrm{~h}$. Each experiment was performed seven times.
After four independent experiments, the attraction zones from $\mathrm{B} 2$ and the mutant complemented with both genes and the gene from $C$. jejuni $81-176$ were $26.5 \pm 2.6 \mathrm{~mm}$, $25 \pm 2.5 \mathrm{~mm}$ and $25.5 \pm 2.6 \mathrm{~mm}$, respectively, whereas the attraction zones of the mutant and the mutant complemented with $c j 0952 c$ or $c j 0951 c$ alone were $10.2 \pm 0.9 \mathrm{~mm}$, $10.0 \pm 1.4 \mathrm{~mm}$ and $10.2 \pm 1.7 \mathrm{~mm}$, as shown in Fig. 6(b). This finding indicates that the $c j 0952 c$ and $c j 0951 c$ genes together encode a receptor that is involved in the chemotactic recognition of formic acid.

\section{The utilization of formic acid is not altered by the presence or absence of Cj0952c-Cj0951C}

Recombinant expression of Cj0952c-Cj0951c and detection of the protein by immunoblotting indicated that both proteins are translated separately and not as a single chemoreceptor. To investigate whether this unusual composition of the chemoreceptor Cj0952c-Cj0951c is related to the utilization of formic acid by formate dehydrogenase, we measured the growth of the wild-type strain B2, the mutant, the cj0952c-cj0951c-complemented mutant and the mutant harbouring the single gene from $C$. jejuni 81-176 in the presence of $10 \mathrm{mM}$ formic acid. As a negative control, the $\mathrm{B} 2$ strain with a transposon insertion in one of the genes encoding the subunits of formate dehydrogenase $(\mathrm{B} 2 \Delta f d h)$ was used. In these experiments, we could not detect a relationship between chemoreceptor presence or composition and formic acid utilization. Wildtype strain B2, the mutant and the complemented mutant showed identical growth, while the growth of $\mathrm{B} 2 \Delta f d h$ was clearly reduced (Fig. 7a). The growth of the same strains in 
(a)
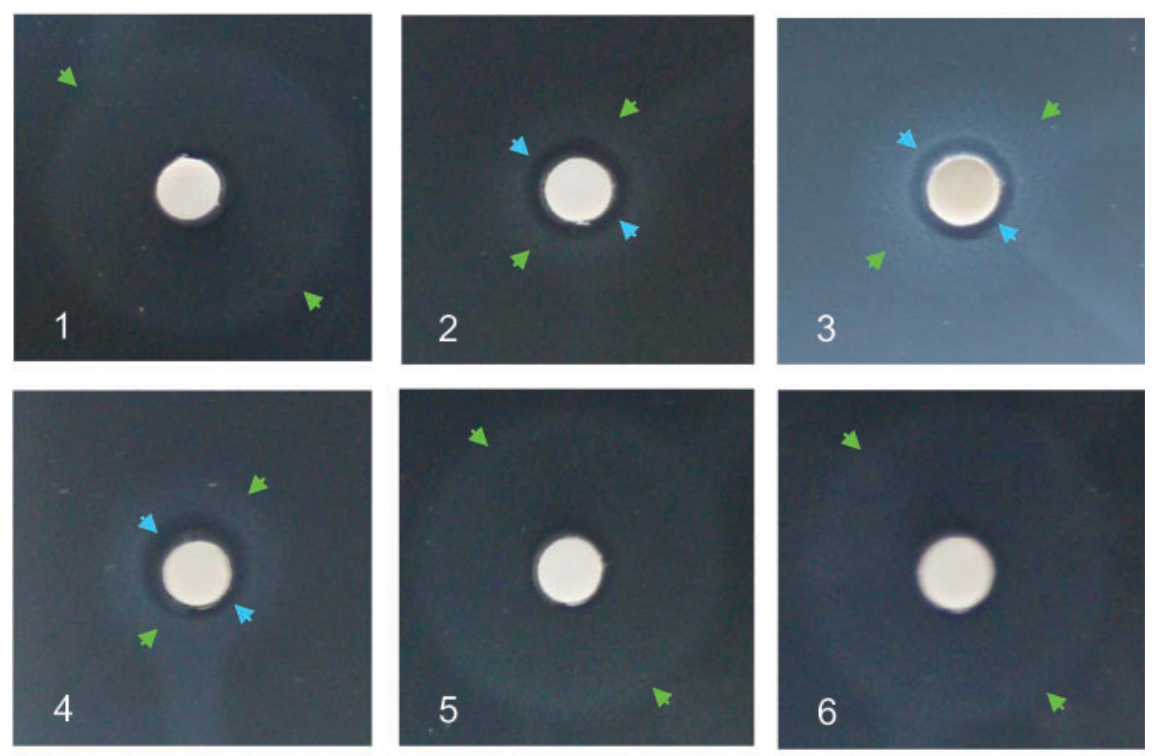

(b)

\begin{tabular}{lcc}
\hline Strain & $\begin{array}{c}\text { Chemoattraction } \\
\text { zone }(\mathrm{mm})\end{array}$ & SD (mm) \\
\hline Wild-type B2 & 26.50 & \pm 2.64 \\
B2 $\Delta$ cj0952c & 10.25 & \pm 0.95 \\
B2 $\Delta$ cj0952c-comp-cj0951c & 10.25 & \pm 1.70 \\
B2 $\Delta$ cj0952c-comp-cj0952c & 10.00 & \pm 1.41 \\
B2 $\Delta$ cj0952c-comp-cj0952c-cj0951c & 25.00 & \pm 2.58 \\
B2 $\Delta$ cj0952c-comp-81-176 & 25.50 & \pm 2.64 \\
\hline
\end{tabular}

\begin{abstract}
Fig. 6. Chemotactic behaviour in the presence of formic acid. 1, Wild-type strain B2; 2, B2 $\Delta c j 0952 c ; 3$, B2 $\Delta$ cj0952c-comp-

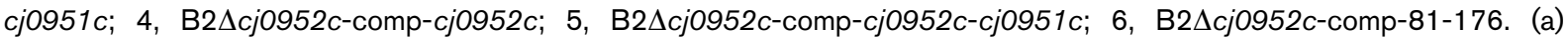
Whereas wild-type strain B2 and the mutant strain B2 $\Delta c j 0952 c$ complemented with cj0952c-cj0951c or the corresponding gene from C. jejuni strain 81-176 showed a significant attraction zone around the formate-soaked paper (green arrows), mutant

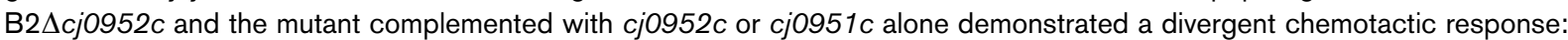
an inner repulsion zone (blue arrow) can be observed surrounded by a dense chemoattractant ring (green arrow). (b) Chemoattractant zones indicated in millimetres. The chemotactic responses of wild-type B2 and the mutant B2 $\Delta$ cj0952c complemented with cj0952c-cj0951c or the respective gene from strain 81-176 were almost identical, whereas cj0952c or cj0951c did not restore the parental phenotype and the complemented mutants showed significantly reduced chemotaxis $(P<0.001)$ towards formic acid.
\end{abstract}

the absence of formic acid gave comparable results, with the exception of the growth of B2 $\Delta f d h$. This was probably due to the presence of a small amount of formic acid in the MH broth which could not be metabolized by B2 $\Delta f d h$ (Fig. 7b).

\section{DISCUSSION}

Since the introduction of transposon mutagenesis techniques to C. jejuni research (Colegio et al., 2001; Golden et al., 2000), this method has proven to be a powerful tool for the identification of many genes related to the pathogenicity of the bacterium. Motility-deficient mutants have been described that have transposon insertions not only in genes with a strong homology to motility genes of known function but also in genes responsible for the chemotactic competence of C. jejuni, e.g. cheA, cheY and cheB (Golden \& Acheson, 2002; Hendrixson et al., 2001). Also, a reporter assay in combination with transposon mutagenesis for the investigation of flagellar regulation has identified genes which are at the beginning of the flagellar transcription cascade (Hendrixson \& DiRita, 2003). Furthermore, a signature-tagged transposon library of $C$. jejuni has detected genes which are involved in the commensal colonization of the gastrointestinal tract of chickens, among them the gene encoding the MCP $\operatorname{docB}$ (Tlp10) and the adjacent gene docA, encoding a putative cytochrome $c$ peroxidase (Hendrixson 
(a)

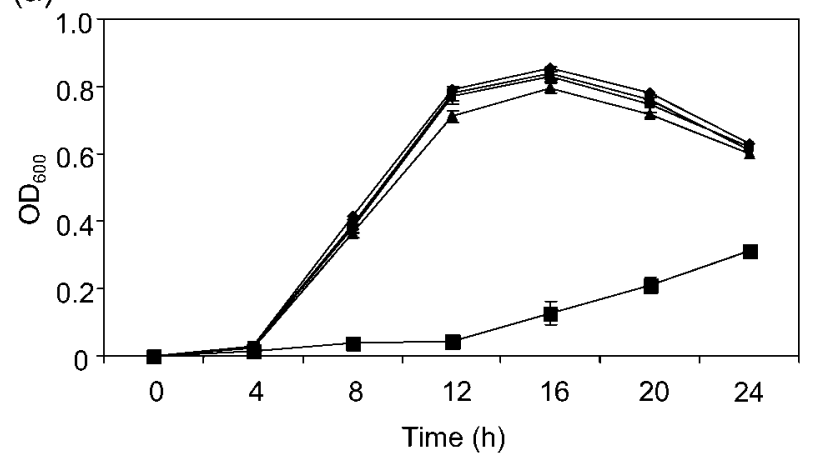

(b)

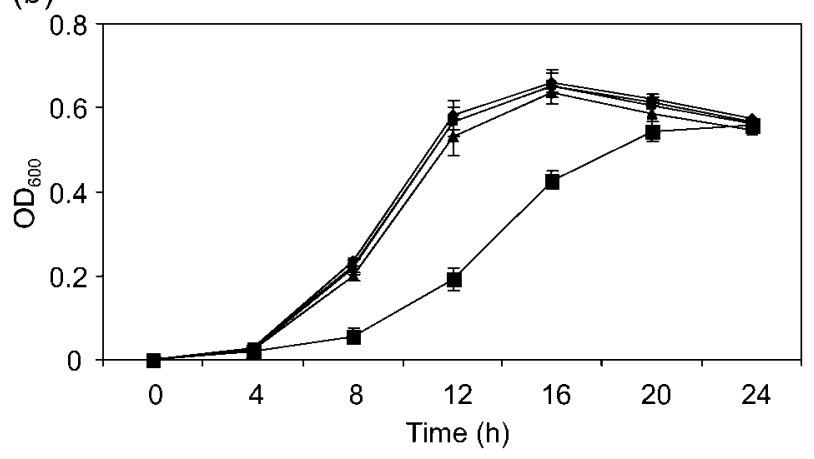

Fig. 7. Growth curves of parental strain B2 ( $), B 2 \Delta c j 0952 c(\boldsymbol{\Delta})$,

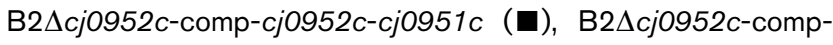
81-176 (O) and $\mathrm{B} 2 \Delta f d h$ (large filled squares) in $\mathrm{MH}$ broth supplemented (a) or not supplemented (b) with $10 \mathrm{mM}$ formic acid. Error bars show SD. See text for details.

\& DiRita, 2004). Signature-tagged transposon mutagenesis has shown Campylobacter colonization in chickens to be complex and dynamic (Grant et al., 2005), and furthermore, screening of a transposon-based mutant library has identified $C$. jejuni genes that are related to polymyxin resistance and to acid adaptation of the pathogen (Lin et al., 2009; Reid et al., 2008). Additionally, transposon mutagenesis in a clinical isolate of $C$. jejuni has detected 26 mutants with a clearly reduced invasion capacity (Javed et al., 2010).

In order to find genes which contribute to the ability of $C$. jejuni to infect Caco-2 cells we screened 660 clones of a transposon-based mutant library of the clinical C. jejuni isolate B2 for altered invasiveness. One of the clones with significant invasion reduction turned out to exhibit transposon insertion in gene cj0952c. C. jejuni gene cj0952c shows similarity to genes encoding MCPs. Depending on the $C$. jejuni strain investigated, cj0952c is thought to be a pseudogene, since the adjacent downstream gene cj0951c encodes a signalling domain, which, together with Cj0952c, would constitute a complete chemoreceptor. Examples of C. jejuni strains in which this chemoreceptor is composed of two single genes are NCTC 11168, CF93-6 and 84-25. Also, in the clinical isolate B2, as in strain NCTC 11168, the two genes are separated. In contrast, in strains 81-176 and 81116 , the corresponding chemoreceptor is encoded by a single gene that encompasses the MCP domain, the transmembrane domain as well as the signalling domain. So far, $c j 0952 c$ has only been described to be upregulated during colonization of the chick caecum (Woodall et al., 2005), although not with regard to its biological function. As for many other bacteria, C. jejuni possesses genes essential for chemotaxis, such as cheA, chew, cheY and MCP receptors. Moreover, chemotactic-mediated motility has been shown to be important for Campylobacter colonization (Takata et al., 1992; Yao et al., 1994). Altogether, 10 genes encoding chemotaxis receptor proteins have been detected in the genome of $C$. jejuni, designated Tlps for transducer-like proteins. These 10 Tlps can further be classified into three different groups (A-C) according to their predicted domain organization (Marchant et al., 2002).

The group A chemoreceptors, to which Cj0952c-Cj0951c (Tlp7) belong, are MCPs and are composed of N-terminal transmembrane domains and a periplasmic ligand-binding domain followed by a HAMP domain and a cytoplasmic signalling domain at the C-terminal end. In this way, the HAMP domain serves as a linker region that is suggested to be conserved in histidine kinases, adenylyl cyclases, methylaccepting chemotaxis proteins and phosphatases, and converts ligand-induced conformational changes into kinase-controlling signals (Aravind \& Ponting, 1999; Butler \& Falke, 1998; Le Moual \& Koshland, 1996; Williams \& Stewart, 1999). Cj0951c shows strong homologies to the cytoplasmic signalling domains of MCPs, being composed of an adaptation region with methylation sites, a flexible region with a conserved glycine hinge, and a protein-interaction region for binding of the chemotaxis protein CheW and the kinase CheA (Hazelbauer et al., 2008).

Although we could clearly demonstrate that transcription of downstream cj0951c was not interrupted, despite the transposon insertion in upstream gene $c j 0952 c$, introduction of cj0952c alone could not restore the parental phenotype. This might be due to the fact that transcription and translation of both genes have to take place at the same time on the same RNA to allow aggregation of both subunits into one functional chemoreceptor.

To confirm $c j 0952 c$ and $c j 0951 c$ to be pseudogenes, which are translated into a single functional chemoreceptor by a read-through mechanism, we cloned both genes in the $C$. jejuni vector pRRC by PCR. For the detection of the in trans-synthesized protein, the $3^{\prime}$ end of the cj0951c primer was expanded by nucleotides encoding a $6 \times$ His-tag. However, immunoblot analysis showed only one protein band of $25 \mathrm{kDa}$, which represents exactly the size of Cj0951c but not the size of a protein composed of Cj0952c and Cj0951c, indicating that Cj0952c and Cj0951c are translated separately in the clinical isolate B2, which corresponds with its genetic composition. Based on our 
findings, at least in C. jejuni strain B2, a non-covalent interaction of both subunits must be postulated for the creation of a functional receptor.

As a prerequisite for infection, $C$. jejuni has to interact with its host. This process is mediated by the chemotaxis system of the pathogen as it guides the pathogen towards the host cells at the respective site of infection. Mutations of the chemotaxis apparatus strongly reduce the ability of $C$. jejuni to invade human epithelial and also chicken cells. Mutant strains that lack cj0019 (Tlp10, docB) or cj0262 (Tlp4, docC) show a decreased colonization of the chicken intestine, and mutagenesis of the chemoreceptors Tlp1 (cj1506), Tlp2 (cj0144), Tlp3 (cj1564), Tlp4 and Tlp10 has demonstrated these receptors to be important for the pathogen to invade host cells (Golden \& Acheson, 2002; Hendrixson \& DiRita, 2004; Vegge et al., 2009). To date, less is known about the chemoreceptors of $C$. jejuni. Recently, Hartley-Tassell et al. (2010) were able to identify Cj1506c (Tlp1) as the aspartate chemosensory receptor of C. jejuni, and thus characterized the first known chemoreceptor of this pathogen responsive to a particular ligand.

In this report we describe how a transposon insertion within $c j 0952 c$ clearly alters the chemotactic behaviour of C. jejuni towards formic acid. While the wild-type is attracted by formic acid, B2 $\Delta c j 0952 c$ shows a biphasic halo with an inner repulsion zone surrounded by a faint ring. The reduced but not completely eliminated attraction towards formic acid might be explained by the energy taxis system of the pathogen. C. jejuni possesses two proteins (CetA, CetB) that together constitute an energy taxis receptor. Thereby, CetB senses changes in the redox state of the electron transport system and transmits this signal to CetA via direct interaction. From CetA, the signal is transduced to the chemotactic machinery, which in turn changes the direction of motility (Hendrixson et al., 2001; Elliott \& DiRita, 2008). Energy taxis has been shown to be an important force driving C. jejuni towards attractants that serve as carbon sources, electron acceptors and, as in the case of formic acid, electron donors (Vegge et al., 2009). The formation of an inner repulsion zone was surprising and might indicate the existence of another chemosensory system that detects formic acid as a chemorepellent. However, when we complemented the mutant with cj0952c-cj0951c or the corresponding gene of C. jejuni strain 81-176, the attraction of B2 towards formic acid could be restored completely.

The genome sequence of $C$. jejuni encodes a formate dehydrogenase which is located on the $f d h A B C D$ operon encompassing the genes cj1511c-cj1508c. Formate dehydrogenase serves as an electron donor enzyme by the oxidation of formate to $\mathrm{CO}_{2}$, protons and electrons. The released electrons, in turn, enter the electron transport chain to contribute to the proton ion gradient (Weerakoon et al., 2009). The discovery that Cj0952c-Cj0951c mediates the chemotaxis of $C$. jejuni in the presence of formic acid raised the question of whether the particular composition of this receptor in the clinical isolate $\mathrm{B} 2$ is related to the utilization of formic acid by formate dehydrogenase. However, growth curves in the presence and absence of formic acid for wild-type strain B2, the mutant B2 $\Delta c j 0952 c$ and the complemented mutant were identical, negating the idea of a combination of the receptor and the enzyme formate dehydrogenase. Interestingly, the presence of formic acid in the medium significantly promoted the growth of all strains (except of B2 $\Delta f d h$ ), indicating that metabolism of formic acid represents an important source of electrons for the pathogen.

The observation that Cj0952c-Cj0951c (Tlp7) in vitro mediates chemotaxis of $C$. jejuni towards formic acid, and that this is related to the infectivity of the pathogen, must now be confirmed in animal models. Experiments are currently under way to investigate this complex within in vivo systems that represent the natural habitat of $C$. jejuni.

\section{ACKNOWLEDGEMENTS}

This work was supported by the Deutsche Forschungsgemeinschaft (GRK 335/3 and GR 906/13-1). We thank A. V. Karlyshev, Kingston University, Kingston upon Thames, UK, and J. E. Galán, Yale University, New Haven, CT, USA, for providing plasmids pRRC and pSB1699.

\section{REFERENCES}

Allos, B. M. (2001). Campylobacter jejuni infections: update on emerging issues and trends. Clin Infect Dis 32, 1201-1206.

Altekruse, S. F., Stern, N. J., Fields, P. I. \& Swerdlow, D. L. (1999). Campylobacter jejuni - an emerging foodborne pathogen. Emerg Infect Dis 5, 28-35.

Aravind, L. \& Ponting, C. P. (1999). The cytoplasmic helical linker domain of receptor histidine kinase and methyl-accepting proteins is common to many prokaryotic signalling domains. FEMS Microbiol Lett 176, 111-116.

Butler, S. L. \& Falke, J. J. (1998). Cysteine and disulfide scanning reveals two amphiphilic helices in the linker region of the aspartate chemoreceptor. Biochemistry 37, 10746-10756.

Colegio, O. R., Griffin, T. J., IV, Grindley, N. D. F. \& Galán, J. E. (2001). In vitro transposition system for efficient generation of random mutants of Campylobacter jejuni. J Bacteriol 183, 2384-2388.

Dasti, J. I., Groß, U., Pohl, S., Lugert, R., Weig, M. \& Schmidt-Ott, R. (2007). Role of plasmid-encoded tet $(\mathrm{O})$ gene in tetracycline-resistant clinical isolates of Campylobacter jejuni and Campylobacter coli. J Med Microbiol 56, 833-837.

Dasti, J. I., Tareen, A. M., Lugert, R., Zautner, A. E. \& Groß, U. (2010). Campylobacter jejuni: a brief overview on pathogenicity-associated factors and disease-mediating mechanisms. Int J Med Microbiol 300, 205-211.

Elliott, K. T. \& DiRita, V. J. (2008). Characterization of CetA and CetB, a bipartite energy taxis system in Campylobacter jejuni. Mol Microbiol 69, 1091-1103.

Everest, P. H., Goossens, H., Butzler, J. P., Lloyd, D., Knutton, S., Ketley, J. M. \& Williams, P. H. (1992). Differentiated Caco-2 cells as a model for enteric invasion by Campylobacter jejuni and C. coli. J Med Microbiol 37, 319-325. 
Friedman, C. R., Neiman, J., Wegener, H. C. \& Tauxe, R. V. (2000), Epidemiology of Campylobacter jejuni in the United States and other industrialized nations. In Campylobacter, 2nd edn, pp. 121-138. Edited by I. Nachamkin \& M. J. Blaser. Washington, DC: American Society for Microbiology.

Golden, N. J. \& Acheson, D. W. (2002). Identification of motility and autoagglutination Campylobacter jejuni mutants by random transposon mutagenesis. Infect Immun 70, 1761-1771.

Golden, N. J., Camilli, A. \& Acheson, D. W. (2000). Random transposon mutagenesis of Campylobacter jejuni. Infect Immun $\mathbf{6 8}$ 5450-5453.

Grant, A. J., Coward, C., Jones, M. A., Woodall, C. A., Barrow, P. A. \& Maskell, D. J. (2005). Signature-tagged transposon mutagenesis studies demonstrate the dynamic nature of cecal colonization of 2week-old chickens by Campylobacter jejuni. Appl Environ Microbiol 71, 8031-8041.

Guerry, P., Ewing, C. P., Hickey, T. E., Prendergast, M. M. \& Moran, A. P. (2000). Sialylation of lipooligosaccharide cores affects immunogenicity and serum resistance of Campylobacter jejuni. Infect Immun 68, 66566662.

Hartley-Tassell, L. E., Shewell, L. K., Day, C. J., Wilson, J. C., Sandhu, R., Ketley, J. M. \& Koroloik, V. (2010). Identification and characterization of the aspartate chemosensory receptor of Campylobacter jejuni. Mol Microbiol 75, 710-730.

Hazelbauer, G. L., Falke, J. J. \& Parkinson, J. S. (2008). Bacterial chemoreceptors: high-performance signalling in network arrays. Trends Biochem Sci 33, 9-19.

Hendrixson, D. R. \& DiRita, V. J. (2003). Transcription of $\sigma^{54}$ dependent but not $\sigma^{28}$-dependent flagellar genes in Campylobacter jejuni is associated with formation of the flagellar secretory apparatus. Mol Microbiol 50, 687-702.

Hendrixson, D. R. \& DiRita, V. J. (2004). Identification of Campylobacter jejuni genes involved in commensal colonization of the chick gastrointestinal tract. Mol Microbiol 52, 471-484.

Hendrixson, D. R., Akerley, B. J. \& DiRita, V. J. (2001). Transposon mutagenesis of Campylobacter jejuni identifies a bipartite energy taxis system required for motility. Mol Microbiol 40, 214-224.

Hugdahl, M. B., Beery, J. T. \& Doyle, M. P. (1988). Chemotactic behaviour of Campylobacter jejuni. Infect Immun 56, 1560-1566.

Javed, M. A., Grant, A. J., Bagnall, M. C., Maskell, D. J., Newell, D. G. \& Manning, G. (2010). Transposon mutagenesis in a hyper-invasive clinical isolate of Campylobacter jejuni reveals a number of genes with potential roles in invasion. Microbiology 156, 1134-1143.

Jin, S., Joe, A., Lynett, J., Hani, E. K., Sherman, P. \& Chan, V. L. (2001). JlpA, a novel surface-exposed lipoprotein specific to Campylobacter jejuni, mediates adherence to host epithelial cells. Mol Microbiol 39, $1225-1236$.

Karlyshev, A. V. \& Wren, B. M. (2005). Development and application of an insertional system for gene delivery and expression in Campylobacter jejuni. Appl Environ Microbiol 71, 4004-4013.

Konkel, M. E., Garvis, S. G., Tipton, S. L., Anderson, D. E., Jr \& Cieplak, W., Jr (1997). Identification and molecular cloning of a gene encoding a fibronectin-binding protein (CadF) from Campylobacter jejuni. Mol Microbiol 24, 953-963.

Konkel, M. E., Kim, B. J., Rivera-Amill, V. \& Garvis, S. G. (1999). Bacterial secreted proteins are required for the internalization of Campylobacter jejuni into cultured mammalian cells. Mol Microbiol 32, 691-701.

Konkel, M. E., Klena, J. D., Rivera-Amill, V., Monteville, M. R., Biswas, D., Raphael, B. \& Mickelson, J. (2004). Secretion of virulence proteins from
Campylobacter jejuni is dependent on a functional flagellar export apparatus. J Bacteriol 186, 3296-3303.

Konkel, M. E., Larson, C. L. \& Flanagan, R. C. (2010). Campylobacter jejuni FlpA binds fibronectin and is required for maximal host cell adherence. $J$ Bacteriol 192, 68-76.

Le Moual, H. \& Koshland, D. E., Jr (1996). Molecular evolution of the C-terminal cytoplasmic domain of a superfamily of bacterial receptors involved in taxis. J Mol Biol 261, 568-585.

Lin, J., Wang, Y. \& Hoang, K. V. (2009). Systematic identification of genetic loci required for polymyxin resistance in Campylobacter jejuni using an efficient in vivo transposon mutagenesis system. Foodborne Pathog Dis 6, 173-185.

Louwen, R., Heikema, A., van Belkum, A., Ott, A., Gilbert, M., Ang, W., Endtz, H. P., Bergman, M. P. \& Nieuwenhuis, E. E. (2008). The sialylated lipooligosaccharide outer core in Campylobacter jejuni is an important determinant for epithelial cell invasion. Infect Immun 76, 4431-4438.

Marchant, J., Wren, B. \& Ketley, J. (2002). Exploiting genome sequences: predictions for mechanisms of Campylobacter chemotaxis. Trends Microbiol 10, 155-159.

Morooka, T., Umeda, A. \& Amako, K. (1985). Motility as an intestinal colonization factor for Campylobacter jejuni. J Gen Microbiol 131, 1973-1980.

Reid, A. N., Pandey, R., Palyada, K., Whitworth, L., Doukhanine, E. \& Stintzi, A. (2008). Identification of Campylobacter jejuni genes contributing to acid adaptation by transcriptional profiling genome-wide mutagenesis. Appl Environ Microbiol 74, 1598-1612.

Schmidt-Ott, R., Pohl, S., Burghard, S., Weig, M. \& Groß, U. (2005). Identification and characterization of a major subgroup of conjugative Campylobacter jejuni plasmids. J Infect 50, 12-21.

Takata, T., Fujimoto, S. \& Amako, K. (1992). Isolation of nonchemotactic mutants of Campylobacter jejuni and their colonization of the mouse intestinal tract. Infect Immun 60, 3596-3600.

Vegge, C. S., Brøndsted, L., Li, Y.-P., Bang, D. D. \& Ingmer, H. (2009). Energy taxis drives Campylobacter jejuni towards the most favourable conditions for growth. Appl Environ Microbiol 75, 5308-5314.

Weerakoon, D. R., Borden, N. J., Goodson, C. M., Grimes, J. \& Olson, J. W. (2009). The role of respiratory donor enzymes in Campylobacter jejuni host colonization and physiology. Microb Pathog 47, 815.

Williams, S. B. \& Stewart, V. (1999). Functional similarities among two-component sensors and methyl-accepting chemotaxis proteins suggest a role for linker region amphipathic helices in transmembrane signal transduction. Mol Microbiol 33, 1093-1102.

Woodall, C. A., Jones, M. A., Barrow, P. A., Hinds, J., Marsden, G. L., Kelly, D. J., Dorrell, N., Wren, B. W. \& Maskell, D. J. (2005). Campylobacter jejuni gene expression in the chick cecum: evidence for adaptation to a low-oxygen environment. Infect Immun 73, 52785285.

Yao, R., Burr, D. H., Doig, P., Trust, T. J., Niu, H. \& Guerry, P. (1994). Isolation of motile and non-motile insertional mutants of Campylobacter jejuni: the role of motility in adherence and invasion of eukaryotic cells. Mol Microbiol 14, 883-893.

Zhang, W., Brooun, A., McCandless, J., Banda, P. \& Adam, M. (1996). Signal transduction in the archeon Halobacterium salinarium is processed through three subfamilies of 13 soluble and membranebound transducer proteins. Proc Natl Acad Sci U S A 93, 4649-4654.

Edited by: P. H. Everest 